\title{
Habilidades Procedimentales del Cálculo Diferencial en el Bachillerato
}

\section{Procedure Skills of Differential Calculus in High School}

Abraham Cuesta-Borges ${ }^{1}$, Beatriz Garza-González ${ }^{2}$ y Heli Herrera-López ${ }^{3}$

Recibido: 16/febrero/2021

Aceptado: $10 /$ abril/2021

Publicado: $16 /$ abril/2021

\section{País}

${ }^{123}$ México

\section{IIII Institución}

${ }^{13}$ Universidad Veracruzana

${ }^{2}$ Universidad Autónoma de Querétaro

\section{$\lambda_{\varnothing}$ Correo Eletrónico}

1cuesta6@hotmail.com

bgarza62@gmail.com

3hherreralopez@hotmail.com

\section{(iD ORCID}

${ }^{1}$ https://orcid.org/0000-0001-9625-2795 ${ }^{2}$ https://orcid.org/0000-0002-5696-6870

${ }^{3} \mathrm{https} / / /$ orcid.org/0000-0003-4257-8794

\section{Citar así: CFAPA / IEEE}

Cuesta-Borges, A., Garza-González, B., \& Herrera-López, H. (2021). Habilidades Procedimentales del Cálculo Diferencial en el Bachillerato. Revista TecnológicaEducativa Docentes 2.0, 11(1), 166-173. https://doi.org/10.37843/rted.v11i1.209

A. Cuesta-Borges, B. Garza-González y H. Herrera-López, "Habilidades Procedimentales del Cálculo Diferencial en el Bachillerato", RTED, vol. 11, n. ${ }^{\circ}$ 1, pp. 166-173, abr. 2021

\section{Resumen}

La educación media superior en México es de vital importancia, debido a ser esta donde el estudiante consolida sus conocimientos en el proceso de selección de los estudios hacia la educación superior. El área fisicomatemática incluye la asignatura de cálculo diferencial, con un nivel elevado de exigencia por la complejidad de otros conceptos de la matemática como variación y representación de modelos matemáticos dentro del estudio de funciones. La presente propuesta consistió en analizar las habilidades procedimentales desarrolladas por los estudiantes en el nivel medio superior al resolver ejercicios de límites y derivación, así como la aplicación del conocimiento en situaciones específicas. En esta investigación se utilizó un enfoque cuantitativo, aplicándose un cuestionario como instrumento orientado a analizar el desempeño de los estudiantes de cálculo diferencial al resolver tanto ejercicios como problemáticas contextualizadas. El estudio se realizó con una población de 40 estudiantes de un bachillerato. De esta manera se determinó si los jóvenes lograron transitar, tanto en los niveles básicos como lo son identificar, resolver, como en aquellos superiores como interpretar o explicar, siendo estos últimos los parámetros necesarios dentro del perfil de egreso del bachillerato.

Palabras clave: Habilidades procedimentales, bachillerato, cálculo diferencial.

\section{Abstract}

Upper secondary education in Mexico is of vital importance because the student consolidates their knowledge in selecting studies towards higher education. The physicalmathematical area includes the subject of differential calculus, with a high level of demand due to the complexity of other mathematics concepts such as variation and representation of mathematical models within the study of functions. The present proposal consisted of analyzing the procedural skills developed by students at the upper secondary level when solving limits and derivation exercises and applying knowledge in specific situations. In this research, a quantitative approach was used, involving a questionnaire to analyze the performance of differential calculus students when solving both exercises and contextualized problems. The study was carried out with a population of 40 high school students. In this way, it was determined whether the young people could travel, both at the basic levels such as identity, solve, and at those higher levels such as interpret or explain, the latter being the necessary parameters within the high school graduation profile.

Keywords: Accompaniment, teaching practices, technology, self-determined learning. 


\section{Introducción}

El bachillerato en México comprende diferentes subsistemas y se divide en Bachillerato General, Bachillerato Tecnológico y la Educación Profesional Técnica (DOF, 2008). El Bachillerato General contempla cuatro componentes de formación propedéutica; éstas conllevan una previa elección, por parte de la estudiante, relacionada con el área de conocimiento próxima a su futura formación profesional. En el área específica de fisicomatemática se incluye la asignatura de cálculo diferencial. Su aprendizaje es primordial, con él los jóvenes del nivel superior pueden tener un buen desempeño en sus primeros semestres universitarios, así como crear procesos de abstracción y variabilidad, elementos necesarios en las carreras de ingeniería y ciencias exactas.

Al visualizar el panorama completo de los diferentes problemas que rodean el bajo desempeño de los estudiantes de cálculo diferencial se han encontrado dos vertientes principales: problemas cognitivos de los estudiantes, así como las estrategias de enseñanza de los docentes. En la primera vertiente se distinguen las dificultades de los jóvenes para trasladar sus habilidades procedimentales hacia problemáticas aplicadas (Prada \& Ramírez, 2017). De igual manera se ha visualizado una carga operativa considerable en los cursos de cálculo diferencial (Sevimli, 2016). Sin embargo, los estudiantes no logran una claridad sobre los significados conceptuales de cada objeto matemático (Bressoud et al., 2016). En ambos casos el efecto ocasionado por las dificultades es un elevado número de reprobación en los cursos de cálculo diferencial.

Por otro lado, al visualizar las estrategias de los docentes, se ha identificado una preferencia por un modelo tradicional de enseñanza (Martínez et al., 2016) donde persiste una mayor prioridad por los procedimientos (Alfaro \& Fonseca, 2019). Bajo este enfoque se ha encontrado, en los cursos de cálculo diferencial, una mayor inclinación hacia los procesos algorítmicos por encima de la dimensión conceptual (López et al., 2018). Ello genera un desajuste dentro de la formación de los jóvenes, quienes deberían generar un aprendizaje incluyente e integrado de cada una de las dimensiones establecidas; es decir, debe existir un componente hilado entre aspectos conceptuales, procedimentales e incluso actitudinales (SEP, 2008).

La importancia de analizar las causas, así como los efectos creados entorno a la enseñanza del cálculo diferencial desde el lugar donde existe el primer acercamiento con la disciplina genera un área de oportunidad para diagnosticar el conjunto de factores que rodean el bajo desempeño de los estudiantes, lo cual permitiría una próxima correlación entre los aportes investigativos previos en conjunto con los nuevos estudios por realizar dentro del bachillerato.

Al analizar las principales problemáticas relativas al bajo desempeño de los estudiantes se ha encontrado un factor común, donde suele existir un mayor desarrollo procedimental de los elementos algebraicos, sin profundizar en una comprensión conceptual. De esta manera las estrategias de enseñanza docentes se encuentran bajo un enfoque de escuela pasiva donde se da un mayor privilegio a la constante repetición de ejercicios, bajo la concepción de propiciar un aprendizaje consolidado y acumulativo a través de tal estrategia (De Zubiria, 1994).

Bajo las nociones previamente descritas se estableció una pregunta de investigación: ¿Qué relación existe entre el adecuado desempeño procedimental de los estudiantes en cálculo diferencial de bachillerato y las dificultades manifiestas para trasladar los aprendizajes hacia situaciones que implican la aplicación del conocimiento? De igual manera se incluye la hipótesis de investigación: Los estudiantes de cálculo diferencial del bachillerato presentan un adecuado desarrollo procedimental algorítmico, pero demuestran dificultades al trasladar los aprendizajes hacia situaciones que implican la aplicación del conocimiento.

A su vez establecido el objetivo general, el cual consistió en: Analizar las habilidades procedimentales y su transición de niveles hacia el desarrollo de situaciones que implican la aplicación del conocimiento en losestudiantes de bachillerato del curso de cálculo diferencial. Los objetivos específicos fueron: i) Determinar las habilidades procedimentales de los estudiantes al resolver ejercicios y situaciones que implican la aplicación del conocimiento en estudiantes de 
bachillerato del curso de cálculo diferencial; ii) Identificar los niveles de resolución que alcanzan los estudiantes al dar solución a ejercicios y a las situaciones que implican la aplicación del conocimiento.

\section{Metodología}

En esta investigación se utilizó un enfoque cuantitativo, aplicándose un cuestionario como instrumento orientado a analizar el desempeño de los estudiantes de cálculo diferencial al resolver tanto ejercicios como problemáticas contextualizadas. Por ejercicios considérese aquellos reactivos donde solo se manejan aspectos procedimentales dentro del curso de cálculo diferencial. Por otro lado, se incluyeron problemáticas donde el estudiante requiere, tanto de habilidades procedimentales como de una conexión conceptual lo cual redunda en una aplicación del conocimiento para brindar una solución a la situación planteada.

El estudio se realizó con una población de 40 estudiantes de un bachillerato los cuales formaban parte del área propedéutica de físico matemáticas, quienes habían finalizado el curso de cálculo diferencial.Se establecieron cuatro secciones en el instrumento: contexto, nociones conceptuales, habilidad procedimental, así como situaciones donde es necesario una aplicación del conocimiento (ver esquema 1).

La primera sección incluyó datos generales de los participantes, como género, edad, así como el conocimiento alcanzado en el curso desde su perspectiva; de igual manera, se indicó el nivel de dificultad estipulado para la asignatura. La segunda sección contiene dos reactivos que permitieron determinar las nociones que tienen los participantes sobre los conceptos básicos del curso, como lo son el límite y la derivada. El tercer apartado estipuló ejercicios de límites, así como derivadas algebraicas, siendo ambas actividades propias de cualquier curso de cálculo diferencial. Dichos reactivos ayudaron no sólo a conocer las habilidades procedimentales, también aportaron un parámetro sobre los niveles de desarrollo alcanzado por los participantes.

El último aparatado planteó dos situaciones de aplicación del conocimiento adquirido a través de problemáticas; éstas permitieron analizar, tanto el desarrollo procedimental como la transición de dichas habilidades hacia un contexto determinado. De esta manera se determinó si los estudiantes establecieron una transición adecuada desde los conocimientos adquiridos dentro de la dimensión procedimental hacia el entorno conceptual, siendo esta relación propia para la resolución de la problemática. Considerando lo establecido por Prada \& Ramírez (2017) es posible establecer la dificultad experimentada por los jóvenes en la consecución de dicho proceso.

\section{Esquema 1}

\section{Secciones del instrumento}

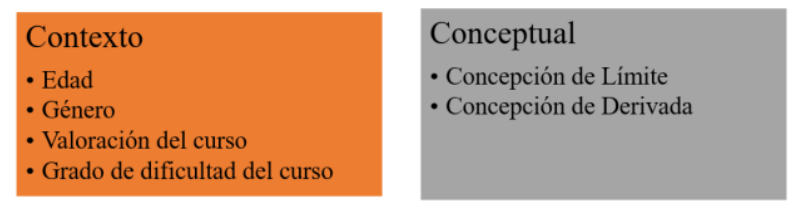

Procedimental -
Algorítmica
- Ejercicios de límites
- Ejercicios de derivación

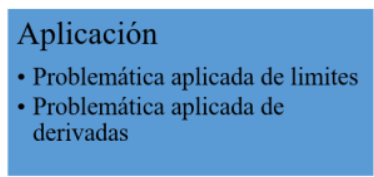

Nota. El esquema muestra cada una de las secciones asignadas en el instrumento, elaboración propia (2021).

El nivel de análisis requería conocer las habilidades desarrolladas, así como las nociones que habían adquirido a lo largo del curso, por ello, los reactivos fueron abiertos con espacio suficiente para el desarrollo de los procedimientos por parte de los participantes. Se aseguró durante la aplicación no existieran circunstancias externas o internas de afectación en su desarrollo, destacando la participación de los jóvenes quienes entregaron sus respuestas con sus hojas de procedimientos. Un factor importante consiste en el cuidado de datos personales, por ello se guardaron las normativas de confidencialidad de la información; de esta manera cada miembro tuvo asignado un código iniciando con la letra $\mathrm{A}$, siendo así el primer participante enlistado como A1 y el último A40.

Las categorías establecidas determinan dos aspectos importantes para esta investigación. Por un lado, en el caso de la sección de ejercicios de límites y de derivación, el proceso de resolución debe visualizar dos aspectos fundamentales; el primero consiste en crear una selección adecuada de la fórmula de derivación o variación (límites) $\mathrm{y}$, cumplido este parámetro, el segundo aspecto consiste en desarrollar algorítmicamente el 
resultado hasta llegar a la respuesta esperada. Considerando esta etapa se analizan cuatros aspectos: 1) Obtención de respuesta esperada, 2) Uso de fórmula adecuada, pero un desarrollo algorítmico deficiente, 3) Uso inadecuado de fórmula, 4) No contestó.

En segunda sección se analizó el desarrollo procedimental de los aspectos donde su maneja la aplicación del conocimiento, se buscaba clarificar la representación creada para resolver el problema; es decir, el vínculo entre las condiciones del problema, las representaciones que utiliza, sus transformaciones, así como sus conversiones (Duval, 1999). Con este fin, se procedió a clasificar el proceso de resolución acorde a dos etapas, primero si el participante planteó la problemática proporcionando un modelo de solución y, logrado este aspecto, si procede a utilizar el modelo a fin de obtener la respuesta esperada. Con base en este proceso, se establecieron cuatro categorías: 1) Obtención de respuesta esperada, 2) Planteamiento y desarrollo de la problemática sin respuesta esperada, 3) Planteamiento de la problemática sin desarrollo y 4) No contestó.

\section{Resultados}

Al analizar esta información se establecen características como: existe prevalencia de hombres por encima de mujeres en ramas cercanas a la matemática, situación planteada por Rojas \& Correa (2014) como elemento común. De igual manera, se resalta la opinión de los participantes quienes consideraron al curso con un nivel bajo de dificultad debido a que más de la mitad consideran a la asignatura como fácil o muy fácil. A su vez, la comprensión de contenidos tuvo un impacto semejante, opciones como muy bueno, excelente abarcaron más de la mitad de los casos de incidencia, en este último aspecto se consideró meramente la opinión de los estudiantes sobre su desempeño en el curso.

Como se comentó, el instrumento se dividió en cuatro secciones las cuales daban información sobre aspectos fundamentales de esta investigación. A continuación, se muestra un resumen de estos aspectos:

\section{Tabla 1}

Contexto de los participantes

Sección 1. Contexto de los participantes

1. Edad promedio de los participantes

\begin{tabular}{ccc}
\hline \multicolumn{2}{c}{17.3 años } \\
\hline 2. & Género & Mujeres \\
\hline Hombres & $\boldsymbol{8}$ \\
\hline
\end{tabular}

3. Desde tu perspectiva. ¿Cuál opción consideras que responde al nivel de dificultad de los contenidos del curso de cálculo diferencial?

\begin{tabular}{lcccc} 
Muy Fácil & Fácil & $\begin{array}{c}\text { Ni fácil } \\
\text { ni } \\
\text { difícil }\end{array}$ & Difícil & Muy difícil \\
\hline 6 & $\mathbf{2 0}$ & $\mathbf{2}$ & $\mathbf{8}$ & $\mathbf{4}$
\end{tabular}

4. Desde tu perspectiva. ¿Cuál opción consideras que responde a tu nivel de comprensión de los contenidos del curso?

\begin{tabular}{ccccc} 
Excelente & $\begin{array}{c}\text { Muy } \\
\text { Bueno }\end{array}$ & Bueno & Suficiente & Deficiente \\
\hline 4 & 18 & 10 & 2 & 6 \\
\hline
\end{tabular}

Nota. Consideraciones del contexto de los participantes y percepción del curso de cálculo diferencial, elaboración propia (2021).

Dentro de la siguiente sección se analizó la comprensión conceptual de los términos de límite y derivada. Al revisar los aspectos propios de la definición, se identificó una baja correlación entre los conceptos donde $60 \%$ de los participantes no tuvieron una noción fundamental de ambos constructos matemáticos. En la gráfica 1 se visualizan los resultados obtenidos.

\section{Gráfica 1}

Nociones conceptuales de los constructos del curso

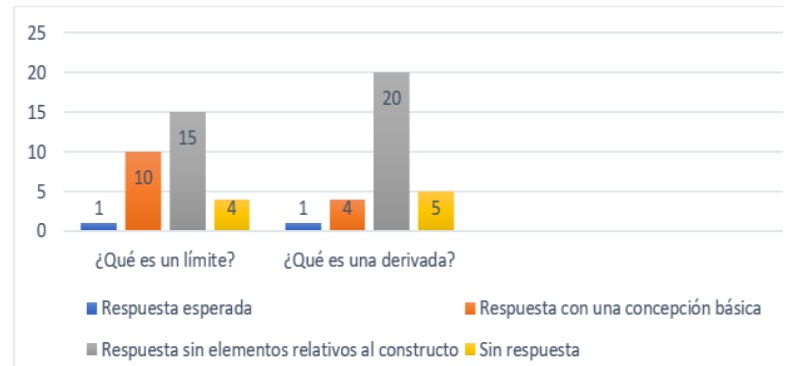

Nota. Percepción de los participantes sobre los conceptos límites - derivadas, elaboración propia (2021).

En el siguiente apartado se analizó el desempeño de los estudiantes al resolver ejercicios de límites, así como derivadas algebraicas, siendo ambos un elemento común dentro de los cursos de cálculo diferencial del bachillerato. El primer bloque incluyó tres límites los cuales se realizaban con métodos de 
factorización previamente abordados como término común, diferencia de cuadrados y descomposición de trinomios. Al ver los resultados, se visualiza (véase la tabla 2) que los jóvenes si logran llegar a la respuesta esperada en los tres ejercicios. Sin embargo, existió un porcentaje considerable de estudiantes quienes no realizaron un desarrollo algebraico o en su defecto, no realizaron ningún procedimiento para dar con la respuesta esperada.

En el segundo bloque se analizó el desempeño de los estudiantes al resolver derivadas con operaciones básicas como lo son suma, resta, multiplicación, división, así como la regla de la cadena. De los cuatro ejercicios propuestos, los participantes mostraron una tendencia semejante a la obtenida en los límites, pero con una tasa menor de incidencia en las respuestas esperadas y en aquellas con un buen desarrollo algebraico. Sin embargo, los participantes sin contestar subieron de manera considerable al duplicarse en reactivos como la regla de la cadena. En las siguientes gráficas se sintetizan los resultados obtenidos.

Tabla 2

Habilidad procedimental

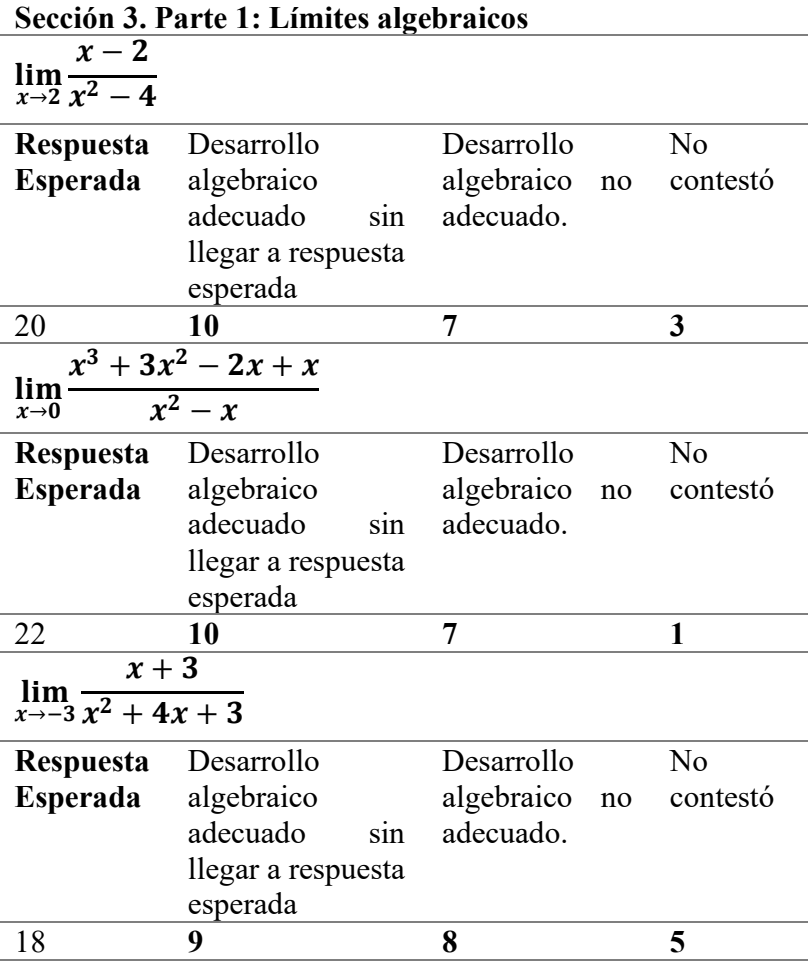

Nota. Clasificación de respuesta de los participantes de acuerdo con la categoría de resolución, elaboración propia (2021).

\section{Gráfica 2}

\section{Habilidad procedimental derivadas}

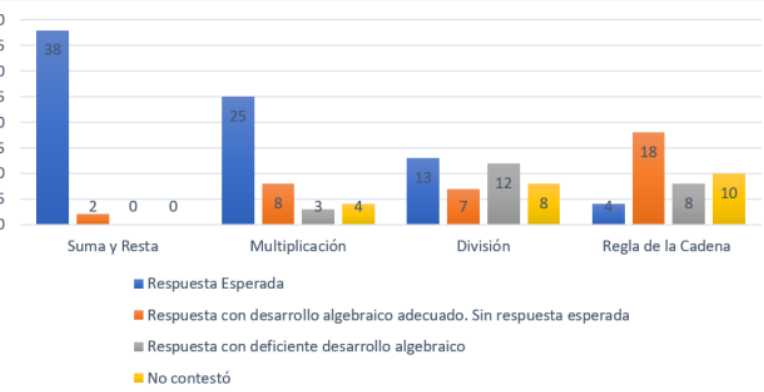

Nota. Nivel de resolución de ejercicios de derivación, elaboración propia (2021).

Por último, la última sección constó de dos situaciones donde se incluía la aplicación del conocimiento; en este bloque los jóvenes debían trasladar toda su habilidad procedimental adquirida y proporcionar una solución a dicho problema. El primer reactivo estipuló incluir por situación el tiro libre realizado por un jugador reconocido de futbol soccer, el cual se visualizaba como una razón de cambio para solicitar su resolución era a través de límites o derivadas. De esta manera era necesario obtener un modelo de desplazamiento del balón a lo largo del tiempo.

Por otro lado, el segundo reactivo consistía en identificar, de manera visual, las concavidades de una función, analizar donde existen máximo y mínimos, así como determinar si son relativos o absolutos. En esta problemática se visualizaba la trayectoria de una partícula a lo largo del tiempo. Esta temática forma parte de la última unidad del curso de cálculo diferencial de bachillerato (Aplicaciones de la derivada). En la siguiente Tabla 3 se visualizan los resultados obtenidos.

\section{Tabla 3}

Aplicación de la derivada

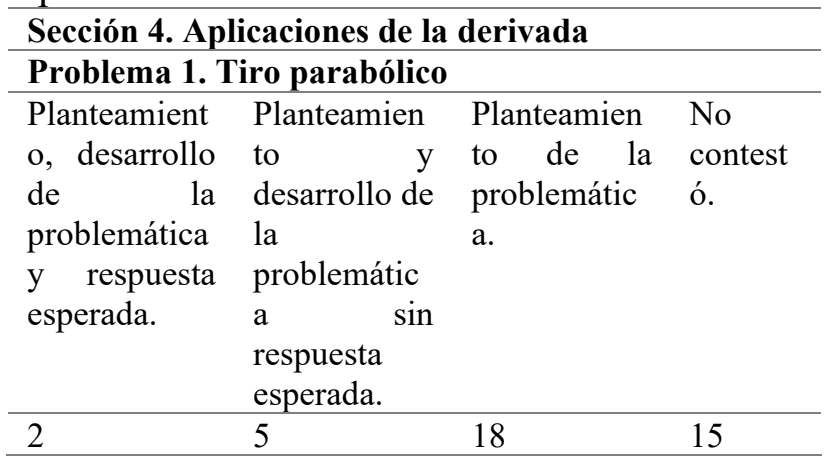




\begin{tabular}{|c|c|c|c|}
\hline \multicolumn{4}{|c|}{ Problema 2. Visualización de concavidades } \\
\hline Identificació & Identificació & No identifica & \\
\hline $\mathrm{n}$ de todas & n parcial de & concavidades, & contest \\
\hline & & pero realiza & ó \\
\hline concavidade & concavidade & procedimiento & \\
\hline S. & S & S. & \\
\hline 4 & 10 & 16 & 10 \\
\hline
\end{tabular}

Nota: Nivel de resolución de problemáticas, elaboración propia (2021).

Con base en los resultados obtenidos en esta última sección se logra ver un menor desempeño en los participantes. En el primer problema se debía derivar una función con sumas o restas, elemento considerado sencillo. Sin embargo, los participantes en su mayoría no lograron llegar a una respuesta esperada. En el segundo caso los jóvenes debían trasladar su concepción de la derivada e identificar las concavidades existentes. (Véase las imágenes 1 y 2). Para este caso, los resultados nuevamente mostraron que más de la mitad de ellos no identificaron de manera adecuada los puntos máximos o mínimos que se solicitaban.

\section{Imagen 1}

Problemática 1 Respuesta Esperada

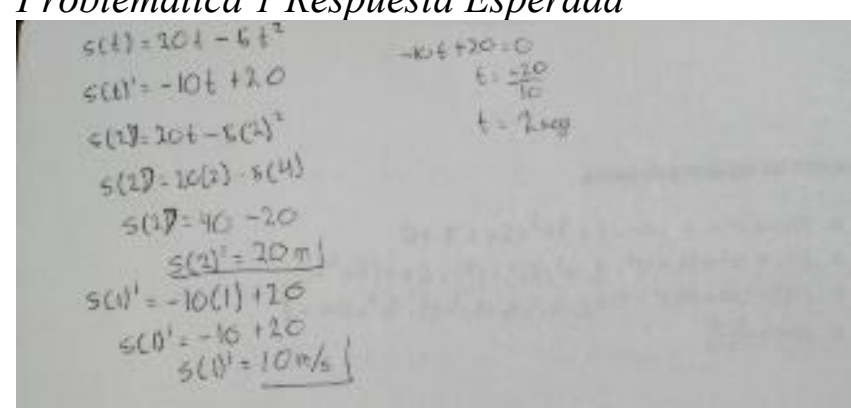

Nota. Respuesta del participante A7, elaboración propia (2021).

\section{Imagen 2}

\section{Problemática 2}

Nota. Respuesta del participante A12, elaborado por los autores, (2021).
Con base en los resultados obtenidos, se encuentra una relación entre lo previamente planteado. No hay una transición entre la habilidad procedimental hacia un entorno de aplicación. De igual manera, la percepción que mantuvieron los estudiantes sobre su desempeño en el curso de cálculo diferencial se encuentra alejada de las habilidades mostradas al resolver tanto los ejercicios como las problemáticas.

\section{Discusión}

Los resultados obtenidos demuestran que los jóvenes tienen un adecuado desarrollo de sus habilidades procedimentales, pero no logran trasladarlas hacia entornos aplicados, lo cual resulta coincidente con lo que Prada \& Ramírez (2017) expone: al resolver problemáticas donde se utilizaba a la derivada los jóvenes no lograban solventarlas. De igual manera se visualizó en los jóvenes poca claridad para definir los conceptos de límite y derivada, lo cual muestra una conexión con la investigación de Bressoud et al., (2016), donde los participantes de igual manera no lograban definir ambos conceptos los cuales son parte del fundamento del curso de cálculo diferencial.

Dentro de los aspectos destacables se encuentra un adecuado manejo procedimental lo cual demuestra un mayor apego a dicha estrategia de enseñanza. Sin embargo, la Reforma Integral a la Educación Media Superior estipula, en su perfil de egreso, que los jóvenes deben tener un desarrollo integral de las dimensiones conceptual, procedimental y actitudinal para trasladar sus aprendizajes a situaciones de su vida cotidiana. En contraste con lo deseado, los resultados del estudio evidencian que no se logra el nexo necesario en el proceso de aprendizaje, tal y como se expone en otra investigación previa (Castro et al., 2017).

Por último, se establece una relación con lo estipulado por López et al., (2018) debido a que los estudiantes no transitan desde un nivel básico de solución, resolviendo ejercicios a través de algoritmos tipificados, hacia un estadio superior como la aplicación del conocimiento adquirido a una problemática en contexto. 


\section{Conclusiones}

Los resultados obtenidos mostraron una vinculación con la pregunta de investigación propuesta, donde los estudiantes de cálculo diferencial del bachillerato presentan un adecuado desarrollo procedimental en el aspecto algebraico, pero demuestran dificultades para trasladar los aprendizajes hacia situaciones aplicadas. Lo cual corrobora la hipótesis inicial de esta investigación.

Bajo esta condición queda claro la importancia de abordar las diferentes problemáticas entorno a la enseñanza del cálculo en el nivel medio superior, que es el nivel educativo donde inicia el estudio de esta asignatura. Se comprende la necesidad de los docentes por seguir un programa de estudios apegado a los contenidos oficiales; sin embargo, es necesaria la implementación de nuevas estrategias y dinámicas apegadas a los lineamientos estipulados por los sistemas educativos de cada nación, tal como lo detalla Escobar \& Nachev (2017). De esta manera resulta necesario plantear rutas de mejora y nuevas áreas de oportunidad, lo cual brindaría una formación temprana de los estudiantes, donde se fomenten $\mathrm{y}$ prioricen conceptos complejos de abstracción - variabilidad. Ello permitiría crear mejores alternativas para evitar problemas cognitivos en los estudiantes y, en consecuencia, coadyuvar a un enfoque de enseñanza centrado en el estudiante.

Otro factor por considerar consiste en la concepción sobre la enseñanza de los docentes, quienes podrían ampliar su panorama de estrategias a través de la incorporación de nuevas herramientas como las Tecnologías de la Información y la Comunicación. La incorporación de dicha tecnología colaboraría al cambio de paradigmas docentes tal como lo establece López quien menciona que ésta se debe concebir como una creación humana en pro de la calidad de vida de los miembros de la sociedad y en favor de una educación que se constituya en parte fundamental del entorno social. A su vez, es importante el apoyo adicional mediante las aplicaciones y software brindadas al proceso de enseñanza tal como Delgado et al., (2020) han propuesto.
Con los datos recabados en esta investigación se abre un área de oportunidad donde se brinde una alternativa a la enseñanza del cálculo desde el nivel medio superior. Dejando un gran panorama para la inclusión de nuevas tecnologías y herramientas dentro de las sesiones, las cuales ayudarán a que los jóvenes tengan una mejor comprensión de los contenidos y al mismo tiempo los docentes cambien su paradigma de enseñanza a través de las nuevas metodologías planteadas por la realidad global y la era tecnológica.

\section{Referencias}

Alfaro, C., \& Fonseca, J. (2019). Propuesta metodológica para la enseñanza del cálculo diferencial e integral en una variable mediante la resolución de problemas para profesores de matemática en formación inicial. CLAME, 32(2). http://funes.uniandes.edu.co/14042/

Bressoud, D., Ghedamsi, I., Martínez, V., \& Törner, G. (2016). Teaching and Learning Calculus. Springer Open. https://www.springer.com/gp/book/9783319329741

Barajas, C., Parada, S., \& Molina, J. (2018). Análisis de dificultades surgidas al resolver problemas de variación. Educación Matemática, 30 (3). http://www.scielo.org.mx/pdf/ed/v30n3/1665-5826ed-30-03-297.pdf

Castro, M., González, M., Flores, S., Ramírez, S., Cruz, M., \& Fuentes, M. (2017). Registros de representación semiótica del concepto de función exponencial. Parte I. Revista entre ciencias. 5 (13). http://dx.doi.org/10.21933/J.EDSC.2017.13.218

Diario Oficial de la Federación. (2008). Acuerdo número 444 por el que se establecen las competencias que constituyen el marco curricular común del Sistema Nacional de Bachillerato. Diario Oficial de la Federación.

De Zubiría, J. (1994). Los modelos pedagógicos. Ministerio de Educación y Cultura de la República de Ecuador. Magisterio.

Delgado, J., Acosta, M., \& Tocto, M. (2020). Experiencia de Diseño de Objeto Virtual de Aprendizaje OVA para Fortalecer el PEA en Estudiantes de Bachillerato. Revista Internacional Tecnológica - Educativa Docentes

2.0. https://ojs.docentes20.com/index.php/revistadocentes20/issue/view/23/38

Duval, R. (1999). Semiosis y pensamiento humano. Registros semióticos de aprendizajes intelectuales. Universidad del Valle.

Escobar, M., \& Nachev, S. (2017). A study of the way five teachers makes decisions in the "EFL Classroom". Colloquia.

http://dspace.uhemisferios.edu.ec:8080/xmlui/bitstre am/handle/123456789/944/PUB\%20ARTICULO\%2 0A\%20study\%20of\%20the\%20way\%20five\%20teac 
hers\%20make\%20decisions\%20in\%20the\%20EFL

$\% 20$ Classroom.pdf? sequence $=1 \&$ is Allowed $=y$

López, C., Aldana, E. \& Erazo, J. (2018). Concepciones de los profesores sobre la resolución de problemas en cálculo diferencial e integral. Revista Logos Ciencia y Tecnología, $\quad 10 \quad$ (1). http://www.redalyc.org/articulo.oa?id=51775445801 1

López, D. (2007). La naturaleza de las tecnologías de información y comunicación: las TIC como determinantes de la organización y de la sociedad de la información. Palabras Clave, $\quad 10 \quad$ (1) http://dspace.uhemisferios.edu.ec:8080/xmlui/bitstre am/handle/123456789/453/1276-5190-1PB.pdf? sequence $=1 \&$ isAllowed $=y$

Martínez, O., Combita, H., \& De la Hoz, E. (diciembre, 2018). Mediación de los Objetos Virtuales de Aprendizaje en el Desarrollo de Competencias Matemáticas en Estudiantes de Ingeniería. Formación Universitaria, 11(6).

https://scielo.conicyt.cl/pdf/formuniv/v11n6/0718 5006-formuniv-11-06-63.pdf

Prada, R., \& Ramírez, P. (2017). Dificultades en la modelización matemática asociadas a la solución de problemas de optimización en cursos de cálculo diferencial. [Acta de Congreso]. VIII Congreso Iberoamericano De Educación Matemática. http://funes.uniandes.edu.co/19594/1/Prada2017Difi cultades.pdf

Rojas, M., \& Correa, D. (2014). ¿El género en las matemáticas? Un análisis de los resultados de las olimpiadas matemáticas. Escenarios, 12(1) https://dialnet.unirioja.es/descarga/articulo/4763430. pdf

Secretaria de Educación Pública. (2008). Competencias que expresan el perfil del docente de la Educación Media Superior. Subsecretaría de educación Media Superior.

Sevimli, E. (2016). Do calculus students demand technology integration into learning environment? case of instructional differences. International Journal of Educational Technology in Higher Education. 13 (1). http://www.redalyc.org/articulo.oa?id=50155029400 2 